\title{
Prevalence of vitamin K and vitamin D deficiency in patients with hepatobiliary and pancreatic disorders
}

\author{
Leon Fisher $^{\mathrm{a}, *}$, Elizabeth Byrnes ${ }^{\mathrm{b}}$, Alexander A. Fisher ${ }^{\mathrm{c}}$ \\ ${ }^{a}$ Department of Gastroenterology, Sir Charles Gairdner Hospital, Nedlands, WA 6009, Australia \\ ${ }^{\mathrm{b}}$ Clinical Biochemistry Research and Development PathWest Laboratory Medicine WA Queen Elizabeth II Medical Centre, Hospital Avenue, Nedlands, \\ WA 6009, Australia \\ ${ }^{\mathrm{c}}$ Division of Medicine, The Canberra Hospital, PO Box 11, Woden, ACT 2606, Australia \\ Received 17 August 2009; revised 8 September 2009; accepted 9 September 2009
}

\begin{abstract}
Keywords:

Abbreviations:

Little is known about the role of fat-soluble vitamins $\mathrm{K}$ and $\mathrm{D}$ in liver function and bone metabolism in biliary and pancreatic diseases associated with cholestasis and/or fat malabsorption. The aim of this study was to determine vitamin $\mathrm{K}$ of bone, vitamin $\mathrm{D}$ and parathyroid hormone status in patients with biliary and pancreatic disorders. In 90 consecutive patients (mean \pm SD age, $65.5 \pm$ 17.7 years; 45 females) undergoing endoscopic retrograde cholangiopancreatography (68 with choledocholithiasis, 14 with other benign condition, and 8 with cholangiopancreatic cancers) fasting concentrations of carboxylated (cOC) and undercarboxylated osteocalcin (ucOC), 25-hydroxyvitamin D, calcium, phosphorus, magnesium, prothrombin time, liver function tests, lipase, and creatinine were measured. Vitamin D deficiency (25-hydroxyvitamin $\mathrm{D}<50 \mathrm{nmol} / \mathrm{L}$ ) was found in $45.6 \%$ of patients and elevated parathyroid hormone levels in $27.8 \%$. The ratio ucOC/cOC (index of vitamin $\mathrm{K}$ deficiency) was above $20 \%$ in $50.6 \%$ of patients, above $30 \%$ in $31 \%$, and above $50 \%$ in $18.4 \%$. Hyperbilirubinemia was a significant independent predictor of low cOC (odds ratio [OR], 11.6; $95 \%$ confidence interval $[\mathrm{CI}], 1.9-59.4 ; P=.07)$. The ratio ucOC/cOC positively correlated with alanine aminotransferase levels $(r=0.410 ; P<.001)$. Elevated $\gamma$-glutamyltransferase $(>180 \mathrm{U} /$ $\mathrm{L})$ and international normalized ratio $(>1.1)$ levels were significant independent predictors of ucOC/ cOC greater than $30 \%$ after adjustment for other covariants $(\mathrm{OR}, 5.5 ; 95 \% \mathrm{CI}, 1.2-25.2 ; P=.027$, and OR, 3.1; 95\% CI, 1.1-8.8; $P=.036$, respectively). This study demonstrates that vitamin $\mathrm{K}$ and vitamin $\mathrm{D}$ deficiencies are common in patients undergoing endoscopic retrograde cholangiopancreatography. Liver dysfunction is associated with and predictive of vitamin $\mathrm{K}$ deficiency of bone and decreased production of osteocalcin, indicating the need for appropriate supplementation. Crown Copyright (C) 2009 Published by Elsevier Inc. All rights reserved.

ERCP; Humans; Osteocalcin; Parathyroid hormone; Vitamin D; Vitamin K

25(OH)D, 25-hydroxyvitamin D; ALP, alkaline phosphatase; ALT, alanine aminotransferase; BMI, body mass index; CLD, chronic liver disease; cOC, carboxylated osteocalcin; CI, confidence interval; CV, coefficient of variation; ERCP, endoscopic retrograde cholangiopancreatography; $\gamma \mathrm{GT}, \gamma$-glutamyltransferase; INR, international normalized ratio; LR, likelihood ratio; OR, odds ratio; PTH, parathyroid hormone; ROC, receiver operating characteristic (ROC); ucOC, undercarboxylated osteocalcin.
\end{abstract}

* Corresponding author. Dr Leon Fisher, 193 Kooyong Road, Caulfield North, VIC Australia. Tel.: +61 3 95050235; fax: +61 395050235.

E-mail address: leonfisher@optusnet.com.au (L. Fisher).

\section{Introduction}

Fat-soluble vitamins $\mathrm{K}$ and $\mathrm{D}$ are important physiologic factors in liver, bone, and vascular metabolism and are powerful regulators of cell proliferation and differentiation [1-4]. 
In higher organisms, vitamin $\mathrm{K}$ is an essential cofactor for $\gamma$-glutamyl carboxylase, an enzyme that catalyses the posttranslational conversion of glutamyl residues into $\gamma$-carboxyglutamate residues in target proteins. In the liver, vitamin $\mathrm{K}$ is responsible for the synthesis of functional forms (eg, carboxylation) of procoagulant factors II, VII, IX, and X as well as anticoagulant proteins $\mathrm{C}, \mathrm{S}$, and $\mathrm{Z}$ [5]. In bone metabolism, the hydroxyapatite binding capacity of osteocalcin (OC), the main noncollagen protein in human bone synthesized exclusively by osteoblasts, is dependent on the degree of vitamin $\mathrm{K}$-mediated carboxylation[6,7]. In case of vitamin $\mathrm{K}$ inadequacy, undercarboxylated $\mathrm{OC}$ (ucOC) is produced. Vitamin $\mathrm{K}$ deficiency of the bone can exist in the absence of vitamin K deficiency of the liver [8]. Serum vitamin $\mathrm{K}$ concentrations fluctuate with recent dietary vitamin $\mathrm{K}$ intake [9] and therefore are not reliable markers of tissue vitamin $\mathrm{K}$ status [10]. Because bones have a high susceptibility to vitamin $\mathrm{K}$ deficiency $[8,11]$, measurement of circulating ucOC levels and especially the ratio between ucOC and cOC (ucOC/cOC) are used widely as sensitive indicators of vitamin $\mathrm{K}$ status of bone [12].

Vitamin $\mathrm{D}$ is metabolized in the liver to 25-hydroxyvitamin D $[25(\mathrm{OH}) \mathrm{D}]$, the main determinant and the best marker of overall vitamin D status [4]. Disorders in vitamin $\mathrm{K}$ and $\mathrm{Ca}$-parathyroid hormone (PTH)-vitamin D status in association with hepatic osteodystrophy have been found in patients with chronic liver diseases (CLDs) [13-19]. However, little is known about these factors in biliary and pancreatic disorders, often associated with transient cholestasis and/or fat malabsorption. Hemorrhagic coagulopathy due to vitamin $\mathrm{K}$-deficient state is recognized in patients with obstructive jaundice, but the skeletal effects of vitamin $\mathrm{K}$ inadequacy are far less clear. The need for vitamin $\mathrm{K}$ and vitamin D supplementation in adults with hepatobiliary and pancreatic disorders has not been fully established.

In this context, the purpose of the present study was to test the hypothesis that patients with biliary and pancreatic disorders undergoing endoscopic retrograde cholangiopancreatography (ERCP) have inadequacy in the vitamin K, vitamin D, and PTH status, might be associated with hyperbilirubinemia and other liver dysfunctions. To our knowledge, this is the first study to determine the prevalence and severity of vitamin $\mathrm{K}$ deficiency of bone, vitamin $\mathrm{D}$, and PTH abnormalities in patients undergoing ERCP and to identify those who may require nutritional supplementation.

\section{Methods and materials}

\subsection{Patient population}

The study population comprised 90 consecutive consenting patients who underwent ERCP at our institution between August 2006 and January 2007. The group consisted of 45 men and 45 women with the mean (SD) age of 65.5 (17.7) years (range, 20-90 years). Complete medical history, including current drug regimen and daily alcohol intake was obtained; physical examination was performed; anthropometric measurements were taken; and body mass index (BMI; weight in kilograms/height in meters squared) was calculated. There were 68 patients with choledocholithiasis, 8 with cholangiopancreatic malignancies (cholangiocarcinoma, 3; pancreatic cancer, 3; ampullary cancer, 1; and metastatic squamous cell carcinoma, 1), 6 with benign biliary strictures, 3 with chronic pancreatitis, 2 with pancreas divisum, 2 with bile leak, and 1 with choledochal cyst. Thirty-five (38.9\%) patients were postcholecystectomy. The diagnosis was based on consistent clinical, laboratory, imaging, and histologic findings. At the time of presentation, 2 of the study patients received vitamin D, 2 received calcium supplements, 2 received bisphosphonates, 1 was on hormone replacement therapy, 1 took prednisolone, 7 took thyroxine, and 3 took ursodeoxycholic acid. Fifteen patients received 5 to $10 \mathrm{mg}$ of vitamin K before ERCP: in 5 subjects because they were taking warfarin and in 10 subjects because of coagulopathy. Statistical analysis was performed before and after exclusion of data from these 15 patients. Most patients $(70$, or $77.8 \%)$ were abstainers or drank minimally (less then $20 \mathrm{~g} / \mathrm{d}$ ). The alcohol consumption before presentation was defined according to Australian alcohol guidelines [20] as moderate if up to $40 \mathrm{~g} / \mathrm{d}$ or $280 \mathrm{~g} / \mathrm{wk}$ in males and up to $20 \mathrm{~g} / \mathrm{d}$ or $140 \mathrm{~g} / \mathrm{wk}$ in females, and as heavy if above these quantities were consumed. Individuals who regularly smoked at least 1 cigarette per day in the last year were classified as current smokers.

This study conformed to the ethical guidelines of Declaration of Helsinki and was approved by the Sir Charles Gairdner Hospital Human Research Ethics Committee. Written informed consent was obtained from all participants.

\subsection{Specimen collection and measurements}

On the day of ERCP fasting, venous samples were obtained and each sample was divided into 2 portions. In 1 portion, hematologic and biochemical parameters including complete blood count, prothrombin time, serum bilirubin, albumin, alanine aminotransferase (ALT), alkaline phosphatase (ALP), $\gamma$-glutamyltransferase $(\gamma \mathrm{GT}), 25(\mathrm{OH}) \mathrm{D}$, intact PTH, calcium, phosphorus, magnesium, lipase, creatinine, and urea nitrogen were determined immediately. Calcium levels were corrected for albumin concentration, and international normalized ratio (INR) and glomerular filtration rate were calculated.

In the other blood portion, sera were separated by centrifugation at $4{ }^{\circ} \mathrm{C}$, frozen, and stored at $-80^{\circ} \mathrm{C}$ until analysis for cOC and ucOC. Both ucOC and cOC were measured with recently developed enzyme immunoassays, which use specific monoclonal antibodies highly reactive to each type (glutamyl or glutamate) of OC (Takara Biomedical Inc, Tokyo, Japan). According to the manufacture, the intraassay and interassay coefficients of variations $(\mathrm{CVs})$ for ucOC were $4.4 \%$ to $6.7 \%$ and $5.7 \%$ to $9.9 \%$, respectively, and for $\mathrm{cOC}$, they were $3.0 \%$ to $4.8 \%$ and $0.7 \%$ to $2.4 \%$, 
respectively. In our laboratory, all CVs were less than $10 \%$. Based on the manufacturer's instructions, the suggested reference range for ucOC is 0.1 to $2.37 \mathrm{ng} / \mathrm{mL}$, and for $\mathrm{cOC}$, it is 1.47 to $10.9 \mathrm{ng} / \mathrm{mL}$.

Serum 25(OH)D concentrations were measured using automated chemiluminescence immunoassay (Dia Sorin Liaison, Stillwater, Minn), and intact serum PTH concentrations were measured by a solid-phase, 2-site chemiluminescent enzyme-labeled immunoassay (Diagnostic Products, Los Angeles, Calif). The intra-assay and interassay CVs were $7 \%$ or less for $25(\mathrm{OH}) \mathrm{D}$ and $8 \%$ or less for PTH.

In the present study, serum $25(\mathrm{OH}) \mathrm{D}$ concentrations were defined as deficient when less than $50 \mathrm{nmol} / \mathrm{L}$, severely moderate deficient when less than $25 \mathrm{nmol} / \mathrm{L}$, insufficient when 50 to $80 \mathrm{nmol} / \mathrm{L}$, and sufficient (adequate) when more than $80 \mathrm{nmol} / \mathrm{L}$ [21]. Based on the manufacturer's data on reference range for PTH, values above $6.8 \mathrm{pmol} / \mathrm{L}$ were defined as hyperparathyroidism.

\subsection{Statistical analyses}

Data are presented as mean values and SDs for continuous normally distributed variables or percentages (with 95\% confidence interval $[\mathrm{CI}]$ ) for categorical variables. Student unpaired $t$ test was used when appropriate (normal data distribution). The difference between more than 2 groups was calculated using 1-way analysis of variance. Statistical analyses included also $\chi^{2}$ test (for categorical variables), Pearson correlation coefficients (for continuous variables), univariate linear correlations, and multivariate logistic regression analyses. Parameters with $P \leq 0.20$ in univariate analysis were included in multivariate model, and manual backwards stepwise logistic regression was performed to determine the best-fitting final model. Goodness of fit was determined by the likelihood ratio $\chi^{2}$ statistic and by the pseudo- $R^{2}$. Receiver operating characteristic (ROC) curves were applied to find the best sensitivity and specificity cutoff value of the variable for the prediction of risk group. In all statistical analyses, 2-tailed tests of significance were used and $P$ values less than .05 were considered statistically significant. For all analyses, Stata, version 10 (Stata Corp, College Station, Tex) was used.

\section{Results}

3.1. Patient characteristics and prevalence of abnormalities in vitamin $K$, vitamin $D$, and PTH status

Demographic and clinical features of the patient population are summarized in Table 1 . Of 90 patients, 50 (55.6\%) were 65 years or older. The subgroup of 75 patients who did not receive warfarin and/or vitamin $\mathrm{K}$ supplementation did not differ from the total group in any of demographic characteristics.

The proportion of all abnormalities in vitamin K, vitamin $\mathrm{D}$, and PTH status is summarized in Table 2. Vitamin D inadequacy $(25(\mathrm{OH}) \mathrm{D}<80 \mathrm{nmol} / \mathrm{L})$ was present in 72 $(80.0 \%)$ of patients. These included $41(45.6 \%)$ patients with vitamin D deficiency, which was severe or moderate in $11(12.2 \%)$ and $31(34.4 \%)$ subjects with vitamin D insufficiency. Secondary hyperparathyroidism occurred in

Table 1

Demographic and clinical characteristics of study patients (total group and by serum bilirubin level)

\begin{tabular}{|c|c|c|c|c|}
\hline \multirow[t]{2}{*}{ Characteristics } & \multirow{2}{*}{$\begin{array}{l}\text { All patients } \\
(\mathrm{n}=90)\end{array}$} & \multicolumn{2}{|c|}{ Serum bilirubin, $\mu \mathrm{mol} / \mathrm{L}$} & \multirow[t]{2}{*}{$P$} \\
\hline & & $<20(\mathrm{n}=46)$ & $\geq 20(\mathrm{n}=44)$ & \\
\hline Age $(y)$, mean \pm SD & $65.5 \pm 17.7$ & $63.8 \pm 19.0$ & $67.3 \pm 16.2$ & .354 \\
\hline Choledocholithiasis, n (\%) & $68(75.6)$ & $35(76.1)$ & $33(75.0)$ & 1.000 \\
\hline Benign stricture, $\mathrm{n}(\%)$ & $6(6.7)$ & $3(6.5)$ & $3(6.8)$ & 1.000 \\
\hline Chronic pancreatitis, n (\%) & $3(3.3)$ & $3(6.5)$ & 0 & .257 \\
\hline \multicolumn{5}{|l|}{ Ethanol consumption } \\
\hline Moderate, n (\%), & $12(13.3)$ & $5(10.9)$ & (15.9) & .694 \\
\hline Heavy, n (\%) & $8(8.9)$ & $3(6.5)$ & $5(11.4)$ & .662 \\
\hline Current smokers, n (\%) & $13(14.4)$ & $6(13.0)$ & $7(15.9)$ & .931 \\
\hline Ex-smokers, n (\%) & $27(30.0)$ & $15(32.6)$ & $12(27.3)$ & .747 \\
\hline \multicolumn{5}{|l|}{ History of } \\
\hline Chronic obstructive pulmonary disease, $\mathrm{n}(\%)$ & $14(15.6)$ & $4(8.7)$ & $10(22.7)$ & .122 \\
\hline Diabetes mellitus, n (\%) & $8(8.9)$ & $6(13.0)$ & $2(4.5)$ & .296 \\
\hline Warfarin use, n (\%) & $5(5.6)$ & $2(4.3)$ & $3(6.8)$ & .960 \\
\hline BMI $\left(\mathrm{kg} / \mathrm{m}^{2}\right)$, mean $\pm \mathrm{SD}$ & $26.9 \pm 5.6$ & $26.6 \pm 5.7$ & $27.1 \pm 5.6$ & .980 \\
\hline
\end{tabular}

Data presented as mean $\pm \mathrm{SD}$ or $\mathrm{n}(\%)$. 
Table 2

Prevalence (\%) of inadequate indices of vitamin D, PTH, and vitamin K of bone status in the total study group and in patients who did not received warfarin and/or vitamin $\mathrm{K}$ (subgroup)

\begin{tabular}{lccccc}
\hline Variables & \multicolumn{2}{c}{$\begin{array}{c}\text { Total group } \\
(\mathrm{n}=90)\end{array}$} & & \multicolumn{2}{c}{$\begin{array}{c}\text { Subgroup } \\
(\mathrm{n}=75)\end{array}$} \\
\cline { 2 - 3 } \cline { 5 - 6 } \cline { 5 - 6 } & Mean & $95 \% \mathrm{CI}$ & & Mean & $95 \% \mathrm{CI}$ \\
\hline $25(\mathrm{OH}) \mathrm{D}(\mathrm{nmol} / \mathrm{L})$ & & & & & \\
$<25$ & 12.2 & $6.3-20.8$ & & 13.3 & 6.6 \\
$25-50$ & 33.4 & $24.4-43.6$ & & 33.4 & 23.7 \\
$50-80$ & 34.4 & $24.7-45.2$ & & 34.7 & 24.0 \\
$>80$ & 20.0 & $12.3-29.8$ & & 18.7 & 10.6 \\
$\mathrm{PTH}>6.8 \mathrm{pmol} / \mathrm{L}$ & 27.8 & $18.9-38.2$ & & 29.7 & $19.7-41.5$ \\
$\mathrm{ucOC} / \mathrm{cOC}(\%)$ & & & & & \\
$>20$ & 50.6 & 39.6 & & 56.2 & 44.1 \\
$>30$ & 31.0 & 21.5 & & 34.2 & 23.5 \\
$>50$ & 18.4 & 10.9 & & 19.2 & 10.9 \\
$25(\mathrm{OH}) \mathrm{D}<50 \mathrm{nmol} /$ and & 16.1 & $9.1-25.5$ & & 17.8 & $9.8-28.5$ \\
ucOC $/ \mathrm{cOC}>30 \%$ & & & & & \\
\hline
\end{tabular}

$25(27.8 \%)$ patients, of whom $16(64 \%)$ had vitamin D deficiency and $5(20 \%)$ vitamin D insufficiency.

The vitamin $\mathrm{K}$ status of bone was defined according to the ratio $\mathrm{ucOC} / \mathrm{cOC}$, expressed as percentage of ucOC concentration to cOC. Increased ucOC/cOC ratio $(\geq 20 \%)$ suggests a poor vitamin $\mathrm{K}$ status. [9,22] The ratio ucOC/cOC was above $20 \%$ in $50.6 \%$ of our patients, above $30 \%$ in $31.0 \%$, and above $50 \%$ in $18.4 \%$, indicating impairment of $\gamma$ carboxylation due to vitamin $\mathrm{K}$ inadequacy in a significant proportion of the study population.

Approximately $16 \%$ of patients demonstrated both vitamin D $(25(\mathrm{OH}) \mathrm{D}<50 \mathrm{nmol} / \mathrm{L})$ and vitamin $\mathrm{K}$ (ucOC/ cOC $>30 \%$ ) deficiency. Vitamin D deficiency was present in $51.9 \%$ of patients with vitamin K deficiency, and vitamin K deficiency was detected in $35 \%$ of subjects with vitamin D deficiency. When analysis was reperformed after exclusion of data from 15 patients who received vitamin $\mathrm{K}$ before ERCP (including 5 patients who were taking warfarin), there were no significant differences in prevalence of inadequacy in vitamin $\mathrm{K}$, vitamin $\mathrm{D}$, or PTH status (Table 2).

\subsection{Effects of hyperbilirubinemia, alcohol, and smoking}

To investigate whether the apparent inadequacy of vitamin $\mathrm{K}$ of bone status and vitamin $\mathrm{D}$ and PTH serum concentrations were associated with liver dysfunction, we subdivided the patients according to their bilirubin level (Tables 1 and 3). All patients with biliary or pancreatic cancer had hyperbilirubinemia ( $>20 \mu \mathrm{mol} / \mathrm{L}$ ), but there were no significant differences between 2 groups in other diagnoses, age, alcohol consumption, smoking habits, comorbid diseases, and BMI. Among patients with hyperbilirubinemia, there was a significant prevalence of males and a lower proportion of subjects who underwent cholecystectomy. Patients with hyperbilirubinemia compared with those with normal serum bilirubin had a marked decrease in both cOC and ucOC, an increase in INR, lower serum phosphorus and, as might be expected, significantly (2.7-4.3 fold) elevated $\gamma \mathrm{GT}$, ALP, and ALT. The groups did not differ in serum ucOC/cOC ratio, concentrations of albumin, $25(\mathrm{OH})$ D, PTH, calcium (corrected), magnesium, lipase, creatinine, hemoglobin levels, or platelet count. Nor were there significant differences between 2 patient groups in the prevalence of abnormalities in the ratio $\mathrm{ucOC} / \mathrm{cOC}, 25(\mathrm{OH})$ D, or PTH levels.

Degree of reduction in $\mathrm{cOC}$ and $\mathrm{ucOC}$ was related to severity of cholestasis. In patients with serum bilirubin greater than $60 \mu \mathrm{mol} / \mathrm{L}$ compared with those with mild hyperbilirubinemia $(20-60 \mu \mathrm{mol} / \mathrm{L}), \mathrm{cOC}(4.9 \pm 5.67$ versus $8.6 \pm 5.04 \mathrm{ng} / \mathrm{mL} ; P=.01)$ and ucOC $(0.9 \pm 0.63$ versus $1.3 \pm 1.04 \mathrm{ng} / \mathrm{mL} ; P=.01)$, levels were significantly lower. When data for the 75 patients who did not receive warfarin and/or vitamin $\mathrm{K}$ were analyzed, these differences in $\mathrm{cOC}$ $(3.8 \pm 3.1$ versus $7.6 \pm 4.2 \mathrm{ng} / \mathrm{mL} ; P=.001)$ and $\mathrm{ucOC}(1.0 \pm$ 0.7 versus $1.5 \pm 1.1 \mathrm{ng} / \mathrm{mL} ; P=.006)$ were also evident.

Multivariate regression analysis with adjustment for age, sex, BMI, 25(OH)D, PTH, INR, and liver function tests revealed that low serum cOC concentration $(<4.2 \mathrm{ng} / \mathrm{mL}$, below the 25th percentile of the distribution in the study group) was significantly and independently associated with hyperbilirubinemia ( $\geq 20 \mu \mathrm{mol} / \mathrm{L}$; odds ratio [OR], 7.1; 95\% CI, 1.2-41.0; $P=.029)$. Other significant and independent predictors of hyperbilirubinemia were elevated $(\times 3) \gamma \mathrm{GT}$ levels $(>180 \mathrm{U} / \mathrm{L})(\mathrm{OR}, 23.3 ; 95 \% \mathrm{CI}, 4.4-122.7 ; P<.001)$,

Table 3

Vitamin $\mathrm{K}$ of bone status, serum $25(\mathrm{OH})$, vitamin D, and PTH concentrations and liver function markers in patients with and without hyperbilirubinemia

\begin{tabular}{|c|c|c|c|}
\hline \multirow[t]{2}{*}{ Parameters } & \multicolumn{2}{|c|}{ Serum bilirubin $(\mu \mathrm{mol} / \mathrm{L})$} & \multirow[t]{2}{*}{$P$} \\
\hline & $<20(\mathrm{n}=46)$ & $\geq 20(\mathrm{n}=44)$ & \\
\hline $\mathrm{cOC}(\mathrm{ng} / \mathrm{mL})$ & $10.8 \pm 7.66$ & $6.9 \pm 6.45$ & .012 \\
\hline $\mathrm{cOC}^{\mathrm{a}}(\mathrm{ng} / \mathrm{mL})$ & $11.1 \pm 7.80$ & $5.6 \pm 4.72$ & .001 \\
\hline $\mathrm{ucOC}, \mathrm{ng} / \mathrm{mL}$ & $2.5 \pm 1.93$ & $1.4 \pm 1.68$ & .007 \\
\hline $\mathrm{ucOC}^{\mathrm{a}}(\mathrm{ng} / \mathrm{mL})$ & $2.6 \pm 1.95$ & $1.2 \pm 0.88$ & .001 \\
\hline $\mathrm{ucOC} / \mathrm{cOC}(\%)$ & $16.1 \pm 19.00$ & $9.6 \pm 6.90$ & .075 \\
\hline $25(\mathrm{OH})$ vitamin $\mathrm{D}(\mathrm{nmol} / \mathrm{L})$ & $57.3 \pm 28.17$ & $53.7 \pm 24.39$ & .522 \\
\hline $\mathrm{PTH}(\mathrm{pmol} / \mathrm{L})$ & $5.6 \pm 2.94$ & $5.6 \pm 3.89$ & .987 \\
\hline Calcium $^{\mathrm{b}}(\mathrm{mmol} / \mathrm{L})$ & $2.4 \pm 0.12$ & $2.4 \pm 0.17$ & .599 \\
\hline Phosphorus (mmol/L) & $1.1 \pm 0.20$ & $1.0 \pm 0.37$ & .049 \\
\hline Magnesium (mmol/L) & $0.82 \pm 0.09$ & $0.81 \pm 0.09$ & .732 \\
\hline Bilirubin $(\mu \mathrm{mol} / \mathrm{L})$ & $10.2 \pm 4.62$ & $108.4 \pm 100.58$ & .000 \\
\hline INR & $1.03 \pm 0.11$ & $1.12 \pm 0.18$ & .005 \\
\hline $\operatorname{ALT}(\mathrm{U} / \mathrm{L})$ & $74.0 \pm 98.91$ & $321.7 \pm 308.62$ & .000 \\
\hline $\operatorname{ALP}(\mathrm{U} / \mathrm{L})$ & $145.9 \pm 84.44$ & $389.8 \pm 323.06$ & .000 \\
\hline$\gamma \mathrm{GT}(\mathrm{U} / \mathrm{L})$ & $167.4 \pm 257.30$ & $622.4 \pm 517.52$ & .000 \\
\hline Albumin (g/L) & $40.7 \pm 4.81$ & $38.7 \pm 5.39$ & .068 \\
\hline Lipase $(\mathrm{g} / \mathrm{L})$ & $61.7 \pm 114.8$ & $232.3 \pm 619.7$ & .076 \\
\hline Creatinine $(\mu \mathrm{mol} / \mathrm{L})$ & $82.1 \pm 26.78$ & $90.3 \pm 29.70$ & .170 \\
\hline Haemoglobin $(g / L)$ & $129.9 \pm 18.6$ & $133.9 \pm 17.9$ & .303 \\
\hline Platelets $\left(\times 10^{9} / \mathrm{L}\right)$ & $300.9 \pm 139.8$ & $265 \pm 92.8$ & .167 \\
\hline
\end{tabular}

Data presented as mean $\pm \mathrm{SD}$.

${ }^{a}$ Patients who did not receive warfarin and/or vitamin $\mathrm{K}$ with serum bilirubin greater than $20 \mu \mathrm{mol} / \mathrm{L}(\mathrm{n}=43)$ and $20 \mu \mathrm{mol} / \mathrm{L}$ or greater $(\mathrm{n}=32)$.

${ }^{\mathrm{b}}$ Corrected for serum albumin concentration. 
ALT levels ( $>120 \mathrm{U} / \mathrm{L} ;$ OR, 4.4; 95\% CI, 1.1-19.0; $P=$ .046 ), and male sex (OR, 8.9; 95\% CI, 1.8-43.7; $P=.007)$.

When we compared patients with moderate or heavy alcohol consumption $(\mathrm{n}=20)$ and subjects with no or mild alcohol intake $(\mathrm{n}=70)$, no significant differences were found in demographic and clinical characteristics nor in biochemical parameters, including serum $\mathrm{cOC}$, ucOC, ucOC/cOC ratio, 25(OH)D, and PTH concentrations (data not shown). Comparison of current smokers $(\mathrm{n}=13)$, ex-smokers $(\mathrm{n}=$ $27)$, and nonsmokers $(n=50)$ revealed only that ex-smokers had a significantly lower ucOC/cOC ratio than non-smokers $(7.9 \pm 5.9 \%$ versus $18.0 \pm 18.5 \% ; P=.022)$.

\subsection{Correlations between cOC, ucOC, 25(OH) D, PTH, and biochemical markers of liver function}

We investigated these interrelationships in 75 patients who did not receive warfarin or vitamin $\mathrm{K}$ (Table 4). Of 18 variables (age, BMI, cOC, ucOC, ucOC/cOC ratio, bilirubin, $\gamma \mathrm{GT}$, ALP, ALT, albumin, INR, 25(OH)D, $\mathrm{PTH}$, calcium, phosphorus, magnesium, creatinine, lipase), serum cOC concentration correlated positively with ucOC and negatively with ratio ucOC/cOC, bilirubin, $\gamma \mathrm{GT}$, and ALT levels. Serum ucOC concentration correlated negatively with bilirubin, $\gamma \mathrm{GT}$, and ALT levels. The ucOC/cOC ratio correlated significantly with ALT levels. As expected, there was a significant positive correlation between bilirubin and $\gamma \mathrm{GT}$, ALP, and ALT levels as well as INR. Serum 25(OH)D concentration was inversely correlated with PTH and BMI, whereas PTH levels correlated positively with serum creatinine, BMI, and age. There was no statistical association between serum 25(OH)D and either OC, biochemical markers of liver function, nor between PTH and these variables.

\subsection{Indicators of vitamin $K$ of bone deficiency, vitamin $D$ deficiency, and low serum cOC}

Using a linear regression model, we also examined the relation between ucOC/cOC more than 30\% (as dependent variable) and liver function markers, serum $25(\mathrm{OH}) \mathrm{D}$ and PTH levels, BMI, age, and sex (as independent variables). By multiple regression analysis, the only significant and independent determinants of the ratio $\mathrm{ucOC} / \mathrm{cOC}$ more than $30 \%$ were elevated $\gamma \mathrm{GT}(>180 \mathrm{U} / \mathrm{L})$ and increased INR $(>1.1)$ as well as cOC values in the lowest quartile and ucOC values in the highest quartile (Table 5). This model correctly classified $81.4 \%$ of patients in the total study group and had sensitivity of $70.3 \%$, specificity of $86.4 \%$, positive predictive value of $70.4 \%$, and negative predictive value of $86.1 \%$. Although sex was not initially significant in multivariate analysis, this became an independent indicator of ucOC/cOC more than $30 \%$ when ucOC and $\mathrm{COC}$ were excluded from the model. In the latter model, male sex had a protective effect (OR, 0.34; 95\% CI, 0.12-0.97; $P=.044$ ) indicating that the effect of sex is captured by absolute levels of cOC and ucOC when these 3 variables compete in the model. 
Table 5

Independent predictors of vitamin $\mathrm{K}$ of bone deficiency, vitamin $\mathrm{D}$ deficiency, and low serum cOC (multivariate analyses ${ }^{a}$ )

\begin{tabular}{llrll}
\hline Dependent variable & Independent variables & OR & $95 \% \mathrm{CI}$ & $P$ \\
\hline ucOC $/ \mathrm{cOC}>30 \%$ & $\gamma \mathrm{GT}>180 \mathrm{U} / \mathrm{L}$ & 5.5 & $1.2-25.2$ & .027 \\
& $\mathrm{INR}>1.1$ & 3.1 & 1.1 & .036 \\
& $\mathrm{cOC}<4.1 \mathrm{ng} / \mathrm{mL}$ & 10.9 & 2.3 & .002 \\
& $\mathrm{ucOC}>2.5 \mathrm{ng} / \mathrm{mL}$ & 5.2 & $1.1-24.3$ & .036 \\
$25(\mathrm{OH}) \mathrm{D}<50 \mathrm{nmol} / \mathrm{L}$ & $\mathrm{PTH}>6.8 \mathrm{pmol} / \mathrm{L}$ & 3.8 & $1.1-11.4$ & .019 \\
$\mathrm{cOC}<4.1 \mathrm{ng} / \mathrm{mL}$ & $\mathrm{Bilirubin}>20 \mu \mathrm{mol} / \mathrm{L}$ & 11.6 & $1.9-59.4$ & .007 \\
& $\mathrm{ucOC} / \mathrm{cOC}>30 \%$ & 10.7 & $1.5-90.5$ & .019 \\
\hline
\end{tabular}

${ }^{a}$ Adjusted for age, sex, liver function markers, BMI, serum 25(OH)D, and PTH levels.

A similar multivariate regression analysis showed that vitamin D deficiency $[25(\mathrm{OH}) \mathrm{D}<50 \mathrm{nmol} / \mathrm{L}]$ was significantly associated only with elevated PTH $(>6.8 \mathrm{pmol} / \mathrm{L})$.

The multivariate regression analysis after adjusting for the above listed covariates also demonstrated that hyperbilirubinemia and $\mathrm{ucOC} / \mathrm{cOC}$ more than $30 \%$ are the only 2 variables independently predictive of low serum cOC (Table 5). The model correctly classified $81.9 \%$ of patients, had a sensitivity of $61.1 \%$ and a specificity of $88.9 \%$, and had a positive predictive value of $64.7 \%$ and a negative predictive value of $87.3 \%$.

An ROC curve was constructed to identify the discriminating value of hyperbilirubinemia in predicting low cOC (Fig. 1). Area under the ROC curve was 0.73. A serum bilirubin level of $20 \mu \mathrm{mol} / \mathrm{L}$ or higher had a sensitivity of $76.2 \%$, a specificity of $61.2 \%$, a positive likelihood ratio $(\mathrm{LR}+)$ of 1.96, and a negative likelihood ratio (LR-) of 0.39 and correctly classified $64.8 \%$ of cases. The predictive value of hyperbilirubinemia was almost optimized when bilirubin level was $37 \mu \mathrm{mol} / \mathrm{L}$ or higher: sensitivity, $71.4 \%$; specificity, 77.6\%; LR+, 3.19; and LR-, 0.37; this threshold correctly classified $76.2 \%$ of cases.

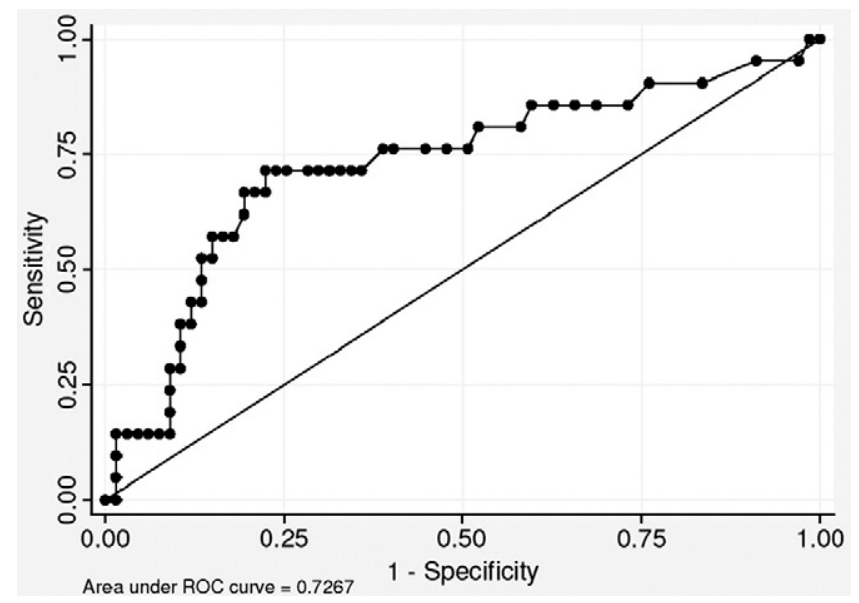

Fig. 1. Receiver operating characteristic curve of serum bilirubin for prediction of low serum $\mathrm{cOC}(<4.1 \mathrm{ng} / \mathrm{mL})$.

\section{Discussion}

The results of this study revealed that a significant proportion of unselected patients with biliary and pancreatic disorders undergoing ERCP has an inadequate vitamin K, vitamin $\mathrm{D}$, and $\mathrm{PTH}$ status and that liver dysfunction is associated with and predictive of vitamin K deficiency of bone and decreased production of OC.

We found subnormal serum levels of $25(\mathrm{OH}) \mathrm{D}$ in $80 \%$ of study patients, vitamin D deficiency in $45.6 \%$, and vitamin D insufficiency in $34.4 \%$. Elevated serum PTH levels were present in $27.8 \%$. The pandemic of vitamin D deficiency is now well recognized [23], and our study population likely reflects this trend. In addition to the common inadequate sunlight exposure and absence of supplementation, in subjects with biliary and pancreatic disorders, malabsorption of vitamin D may also contribute to vitamin D insufficiency or deficiency.

Vitamin D deficiency affects the immune system, cell growth, and differentiation; increases production of proinflammatory cytokines and acute-phase proteins; and increases the risk of cancer [24]. Secondary hyperparathyroidism also significantly promotes acute-phase responses. It is possible that vitamin D inadequacy and PTH elevation observed in our patients were among factors that determined the severity of the inflammatory and malignant processes.

We used ucOC/cOC ratio as a surrogate marker for vitamin $\mathrm{K}$ of bone status. The rationale behind this approach is that this ratio (an estimate of the degree of carboxylation of circulating OC) is independent of bone turnover and, therefore, is a more reliable marker of tissue stores of vitamin $\mathrm{K}$ than serum ucOC, which was often used as an indicator of vitamin status of the bone. In our study group, ucOC/cOC was above $20 \%$ in $50.6 \%$ of patients, above $30 \%$ in $31.0 \%$, and above $50 \%$ in $18.4 \%$. In young healthy persons, $92 \%$ of circulating OC is carboxylated, and this increases to $98 \%$ with dietary phylloquinone supplementation [25]. The median percentage of ucOC in healthy girls was $13.6 \%$ in one study [10] and $21.9 \%$ in another [12]. A cutoff point of $20 \%$ or higher for $\mathrm{ucOC} / \mathrm{cOC}$ ratio was selected as suggestive of vitamin $\mathrm{K}$ deficiency in adults $[9,22]$. Taking into account that the percentage of ucOC measured by immunosorbent assays is higher compared with its measurement by hydroxyapatite assays [26], we used ucOC/cOC more than $30 \%$ as an indicator of vitamin $\mathrm{K}$ inadequacy.

In our cohort, vitamin D deficiency was present in $45.6 \%$ of patients and vitamin $\mathrm{K}$ inadequacy in $31.0 \%$, but both deficiencies were seen only in $16 \%$, suggesting a different cause in individual patients. Low serum $25(\mathrm{OH}) \mathrm{D}$ concentrations negatively correlated with elevated ucOC and ucOC/ cOC ratio in older women with hip fracture [22] and in adolescent girls [12]. However, we found no significant relationship between serum 25(OH)D nor PTH levels and indicators of vitamin $\mathrm{K}$ status. This shows that vitamin $\mathrm{K}$ status was not directly influenced by the vitamin D-PTH 
axis. The possible causes of vitamin $\mathrm{K}$ deficiency in patients with hepatopancreatobiliary disease, in addition to poor dietary intake, include decreased vitamin $\mathrm{K}$ absorption from intestine, disturbance of vitamin $\mathrm{K}$ cycle, and the decrease in vitamin $\mathrm{K}$ storage. In agreement with previous reports $[27,28]$, our study did not show a significant influence of long-term therapeutic anticoagulation with warfarin and/or short-term vitamin K supplementation on markers of vitamin $\mathrm{K}$ status of bone. In line with other studies [29], we also found that more patients were vitamin $\mathrm{K}$ insufficient in bone than in liver as ucOC/cOC more than $30 \%$ was found in $34.2 \%$, whereas INR more than 1.1 (in patients not receiving warfarin) was found only in $8.9 \%$.

In this study, there was no association found between inadequate vitamin $\mathrm{K}$ status and diagnosis, age, or alcohol consumption; male sex demonstrated a protective effect; and ex-smokers had a lower mean $\mathrm{ucOC} / \mathrm{cOC}$ ratio than did nonsmokers. These data are consistent with studies that have shown no relationship between specific diagnosis and osteodystrophy in CLD [15], between plasma phylloquinone concentrations and alcohol consumption in the general population [9], and higher plasma phylloquinone levels in men than in women [30]. There are reports of agerelated increase in vitamin $\mathrm{K}$ requirements, especially in postmenopausal women [27], and the majority of our patients were in the fifth to seventh decades of life (mean age was 65 years). Patients who quit smoking possibly increased the dietary intake of vitamin $\mathrm{K}$ as a result of changed lifestyle.

In the present study, the ratio ucOC/cOC positively correlated with ALT levels, a sensitive marker of hepatocellular damage. Furthermore, elevated $\gamma \mathrm{GT}(>180 \mathrm{U} / \mathrm{L})$ and INR $(>1.1)$ levels were significant and independent predictors of ucOC/cOC more than $30 \%$ after adjustment for other covariants, indicating a significant relationship between liver dysfunction and vitamin $\mathrm{K}$ status. It is well known that absorption of vitamin $\mathrm{K}$ from the proximal intestine is dependent on bile and pancreatic juice secretion. In patients with obstructive jaundice, approximately $80 \%$ of radioisotopic vitamin $\mathrm{K}$ is excreted unaltered compared with $20 \%$ in healthy subjects $[31,32]$. It seems, therefore, that extrahepatic cholestasis, as in our patients, is a very important contributor to vitamin $\mathrm{K}$ insufficiency, which in turn causes further progression of liver dysfunction manifesting in elevated INR, ALT, and $\gamma$ GT. A defect in vitamin $\mathrm{K}-$ dependent prothrombin carboxylation affects the coagulation mechanism, and the presence of undercarboxylated prothrombin is also a well-recognized marker for hepatocellular carcinoma with tumor growth promoting properties [33].

An important finding in our study was the strong association of hyperbilirubinemia and other markers of liver dysfunction with depressed osteoblastic function as reflected in the synthesis of OC and, consequently, low serum $\mathrm{COC}$ and ucOC levels. Patients with hyperbilirubinemia had significantly lower mean values of cOC and ucOC than patients with normal serum bilirubin, and these variables demonstrated a dose-response relation. There was also a significant inverse correlation between serum cOC as well as ucOC concentrations and bilirubin, $\gamma \mathrm{GT}$, and ALT levels. Moreover, hyperbilirubinemia and vitamin $\mathrm{K}$ inadequacy (ucOC/cOC $>30 \%$ ) were the only 2 variables associated independently with low serum cOC concentrations, and the latter could be predicted from these variables with an accuracy of more than $81 \%$. These data suggest that cholestasis, along with vitamin $\mathrm{K}$ deficiency, may be a major pathophysiologic factor affecting OC production, an informative marker of osteoblast activity. Our findings are consistent with several but not all previous studies. In patients with various CLD, serum bilirubin correlated with OC [34], bone mineral density [35], and bone loss [36,37]. Serum OC levels in cirrhotic patients compared with controls were reported to be lower [34,38,39]. However, some studies did not confirm the association between serum bilirubin and bone mineral density in CLD patients [17,40]. A dose-related toxic effect of bilirubin on osteoblast proliferation was shown in vitro studies with plasma taken from patients with jaundice due to different causes [41].

Most published studies like ours suggest that cholestasis contributes substantially to OC synthesis. The findings of this study emphasize the link between extrahepatic cholestasis, suboptimal vitamin $\mathrm{K}$ status, and OC production. Low OC production, high ucOC/cOC ratio, and vitamin D insufficiency, all three are recognized markers of altered bone homeostasis and independent risk factors for fracture. It should also be noted that skeleton through secretion of OC exerts a profound and complex influence on glucose and fat metabolism [42]. The high prevalence of poor vitamin $\mathrm{K}$ and vitamin $\mathrm{D}$ status, 2 factors with pleiotropic actions and of vital importance for liver, bone, and vascular health, indicate that corrective strategies should be considered as a part of "standard of care" in patients undergoing ERCP. Unfortunately, this is often ignored by practicing physicians.

This study has several limitations. First, because of its cross-sectional design, the causative nature of the associations cannot be established. Second, to determine the beneficial effects of supplementation with vitamin $\mathrm{K}$, vitamin $\mathrm{D}$, and calcium and the optimal doses needed, it will be necessary to perform a randomized controlled trial.

In conclusion, our study suggests that inadequate vitamin $\mathrm{K}$ and vitamin $\mathrm{D}$ status should be anticipated in patients undergoing ERCP. Liver dysfunction is predictive for vitamin $\mathrm{K}$ deficiency of bone, and cholestasis is associated with depressed OC production. Further studies are needed to elucidate therapeutic benefits of vitamin D and vitamin $\mathrm{K}$ supplementation in these patients.

\section{Acknowledgment}

We would like to thank the Research Fund of Sir Charles Gairdner Hospital, Perth, Australia, for providing a grant for this study. 


\section{References}

[1] Cranenburg EC, Schurgers LJ, Vermeer C. Vitamin K: the coagulation vitamin that became omnipotent. Thromb Haemost 2007;98:120-5.

[2] Kaneki M, Hosoi T, Ouchi Y, Orimo H. Pleiotropic actions of vitamin $\mathrm{K}$ : protector of bone health and beyond? Nutrition 2006;22:845-52.

[3] Lips P. Vitamin D physiology. Prog Biophys Mol Biol 2006;92:4-8.

[4] Holick MF. Vitamin D deficiency. N Engl J Med 2007;357:266-81.

[5] Nelsestuen GL, Shah AM, Harvey SB. Vitamin K-dependent proteins. Vitam Horm 2000;58:355-89.

[6] Koshihara Y, Hoshi K. Vitamin K2 enhances osteocalcin accumulation in the extracellular matrix of human osteoblasts in vitro. J Bone Miner Res 1997;12:431-8.

[7] Iwamoto J, Sato Y, Takeda T, Matsumoto H. High-dose vitamin K supplementation reduces fracture incidence in postmenopausal women: a review of the literature. Nutr Res 2009;29:221-8.

[8] Vermeer C, Knapen MH, Schurgers LJ. Vitamin K and metabolic bone disease. J Clin Pathol 1998;51:424-6.

[9] McKeown NM, Jacques PF, Gundberg CM, Peterson JW, Tucker KL, Kiel DP, et al. Dietary and nondietary determinants of vitamin K biochemical measures in men and women. J Nutr 2002;132:1329-34.

[10] Kalkwarf HJ, Khoury JC, Bean J, Elliot JG. Vitamin K, bone turnover, and bone mass in girls. Am J Clin Nutr 2004;80:1075-80.

[11] Shearer MJ. Vitamin K. Lancet 1995;345:229-34.

[12] O'Connor E, Molgaard C, Michaelsen KF, Jakobsen J, LambergAllardt CJ, Cashman KD. Serum percentage undercarboxylated osteocalcin, a sensitive measure of vitamin $\mathrm{K}$ status, and its relationship to bone health indices in Danish girls. Br J Nutr 2007; 97:661-6.

[13] Collier JD, Ninkovic M, Compston JE. Guidelines on the management of osteoporosis associated with chronic liver disease. Gut 2002;50 (Suppl 1):i1-i9.

[14] Shiomi S, Nishiguchi S, Kubo S, Tamori A, Habu D, Takeda T, et al. Vitamin K2 (menatetrenone) for bone loss in patients with cirrhosis of the liver. Am J Gastroenterol 2002;97:978-81.

[15] Leslie WD, Bernstein CN, Leboff MS. AGA technical review on osteoporosis in hepatic disorders. Gastroenterology 2003;125:941-66.

[16] Targher G, Bertolini L, Scala L, Cigolini M, Zenari L, Falezza G, et al. Associations between serum 25-hydroxyvitamin D3 concentrations and liver histology in patients with non-alcoholic fatty liver disease. Nutr Metab Cardiovasc Dis 2007;17:517-24.

[17] Smith DL, Shire NJ, Watts NB, Schmitter T, Szabo G, Zucker SD. Hyperbilirubinemia is not a major contributing factor to altered bone mineral density in patients with chronic liver disease. J Clin Densitom 2006;9:105-13.

[18] Mager DR, McGee PL, Furuya KN, Roberts EA. Prevalence of vitamin $\mathrm{K}$ deficiency in children with mild to moderate chronic liver disease. J Pediatr Gastroenterol Nutr 2006;42:71-6.

[19] Fisher L, Fisher A. Vitamin D and parathyroid hormone in outpatients with noncholestatic chronic liver disease. Clin Gastroenterol Hepatol 2007;5:513-20.

[20] National Health and Medical Research Council (Australia). Australian alcohol guidelines : health risks and benefits. Canberra: National Health and Medical Research Council; 2001. p. vi, 128.

[21] Vitamin D and adult bone health in Australia and New Zealand: a position statement. Med J Aust 2005;182:281-5.

[22] Szulc P, Chapuy MC, Meunier PJ, Delmas PD. Serum undercarboxylated osteocalcin is a marker of the risk of hip fracture in elderly women. J Clin Invest 1993;91:1769-74.

[23] Plehwe WE. Vitamin D deficiency in the 21st century: an unnecessary pandemic? Clin Endocrinol (Oxf) 2003;59:22-4.
[24] Holick MF. High prevalence of vitamin D inadequacy and implications for health. Mayo Clin Proc 2006;81:353-73.

[25] Binkley NC, Krueger DC, Kawahara TN, Engelke JA, Chappell RJ, Suttie JW. A high phylloquinone intake is required to achieve maximal osteocalcin gamma-carboxylation. Am J Clin Nutr 2002;76:1055-60.

[26] Gundberg CM, Nieman SD, Abrams S, Rosen H. Vitamin K status and bone health: an analysis of methods for determination of undercarboxylated osteocalcin. J Clin Endocrinol Metab 1998;83: 3258-66.

[27] Tsugawa N, Shiraki M, Suhara Y, Kamao M, Tanaka K, Okano T. Vitamin $\mathrm{K}$ status of healthy Japanese women: age-related vitamin $\mathrm{K}$ requirement for gamma-carboxylation of osteocalcin. Am J Clin Nutr 2006;83:380-6.

[28] Binkley N, Krueger D, Engelke J, Suttie J. Vitamin K deficiency from long-term warfarin anticoagulation does not alter skeletal status in male rhesus monkeys. J Bone Miner Res 2007;22:695-700.

[29] Kuwabara A, Tanaka K, Tsugawa N, Nakase H, Tsuji H, Shide K, et al. High prevalence of vitamin K and D deficiency and decreased BMD in inflammatory bowel disease. Osteoporos Int 2009;20:935-42.

[30] Thane CW, Wang LY, Coward WA. Plasma phylloquinone (vitamin K1) concentration and its relationship to intake in British adults aged 19-64 years. Br J Nutr 2006;96:1116-24.

[31] Shearer MJ, McBurney A, Barkhan P. Studies on the absorption and metabolism of phylloquinone (vitamin K1) in man. Vitam Horm 1974; 32:513-42.

[32] Barkhan P, Shearer MJ. Metabolism of vitamin K1 (phylloquinone) in man. Proc R Soc Med 1977;70:93-6.

[33] Carr BI, Kar S, Wang M, Wang Z. Growth inhibitory actions of prothrombin on normal hepatocytes: influence of matrix. Cell Biol Int 2007;31:929-38.

[34] Diamond T, Stiel D, Mason R, Lissner D, Bikle D, Wilson S, et al. Serum vitamin D metabolites are not responsible for low turnover osteoporosis in chronic liver disease. J Clin Endocrinol Metab 1989; 69:1234-9.

[35] Eastell R, Dickson ER, Hodgson SF, Wiesner RH, Porayko MK, Wahner HW, et al. Rates of vertebral bone loss before and after liver transplantation in women with primary biliary cirrhosis. Hepatology 1991;14:296-300.

[36] Menon KV, Angulo P, Weston S, Dickson ER, Lindor KD. Bone disease in primary biliary cirrhosis: independent indicators and rate of progression. J Hepatol 2001;35:316-23.

[37] Ormarsdottir S, Ljunggren O, Mallmin H, Michaelsson K, Loof L. Increased rate of bone loss at the femoral neck in patients with chronic liver disease. Eur J Gastroenterol Hepatol 2002;14:43-8.

[38] Monegal A, Navasa M, Guanabens N, Peris P, Pons F, Martinez de Osaba MJ, et al. Osteoporosis and bone mineral metabolism disorders in cirrhotic patients referred for orthotopic liver transplantation. Calcif Tissue Int 1997;60:148-54.

[39] Trautwein C, Possienke M, Schlitt HJ, Boker KH, Horn R, Raab R, et al. Bone density and metabolism in patients with viral hepatitis and cholestatic liver diseases before and after liver transplantation. Am J Gastroenterol 2000;95:2343-51.

[40] Newton J, Francis R, Prince M, James O, Bassendine M, Rawlings D, et al. Osteoporosis in primary biliary cirrhosis revisited. Gut 2001;49: 282-7.

[41] Janes CH, Dickson ER, Okazaki R, Bonde S, McDonagh AF, Riggs BL. Role of hyperbilirubinemia in the impairment of osteoblast proliferation associated with cholestatic jaundice. J Clin Invest 1995; 95:2581-6.

[42] Lee NK, Karsenty G. Reciprocal regulation of bone and energy metabolism. Trends Endocrinol Metab 2008;19:161-6. 